\title{
Neuregulin-1: An underlying protective force of cardiac dysfunction in sepsis (Review)
}

\author{
WEN KANG $^{1}$, YUE CHENG ${ }^{2}$, XI WANG ${ }^{2}$, FANG ZHOU $^{1}$, \\ CHENLIANG ZHOU ${ }^{3}$, LONG WANG ${ }^{1}$ and LIANG ZHONG ${ }^{4}$ \\ Departments of ${ }^{1}$ Anesthesiology, ${ }^{2}$ Cardiology and ${ }^{3}$ Critical Care Medicine, Renmin Hospital \\ of Wuhan University; ${ }^{4}$ Department of Anesthesiology, Wuhan Medical and Healthcare Center \\ for Women and Children, Wuhan, Hubei 430060, P.R. China
}

Received April 6, 2019; Accepted March 4, 2020

DOI: $10.3892 / \mathrm{mmr} .2020 .11034$

\begin{abstract}
Neuregulin-1 (NRG-1) is a type of epidermal growth factor-like protein primarily distributed in the nervous and cardiovascular systems. When sepsis occurs, the incidence of cardiac dysfunction in myocardial injury is high and the mechanism is complicated. It directly causes myocardial cell damage, whilst also causing damage to the structure and function of myocardial cells, weakening of endothelial function and coronary microcirculation, autonomic dysfunction, and activation of myocardial inhibitory factors. Studies investigating NRG-1 have been performed using a variety of methods, including in vitro models, and animal and human clinical trials; however, the results are not consistent. NRG-1/ErbBs signaling is involved in a variety of cardiac processes, from the development of the myocardium and cardiac conduction systems to the promotion of angiogenesis in cardiomyocytes, and in cardio-protective effects during injury. NRG-1 may exert a multifaceted cardiovascular protective effect by activating NRG-1/ErbBs signaling and regulating multiple downstream signaling pathways, thereby improving myocardial cell dysfunction in sepsis, and protecting cardiomyocytes and endothelial cells. It may alleviate myocardial microvascular endothelial injury in sepsis; its anti-inflammatory effects inhibit the production of myocardial inhibitory factors in sepsis, improve myocardial ischemia, decrease oxidative stress, regulate the disruption to the homeostasis of the autonomic nervous system, improve diastolic function, and offer protective effects at multiple target sites. As
\end{abstract}

Correspondence to: Professor $\mathrm{Xi}$ Wang, Department of Cardiology, Renmin Hospital of Wuhan University, 9 Zhangzhidong Road, Wuhan, Hubei 430060, P.R. China

E-mail: xiwangwhu@163.com

Professor Chenliang Zhou, Department of Critical Care Medicine, Renmin Hospital of Wuhan University, 9 Zhangzhidong Road, Wuhan, Hubei 430060, P.R. China

E-mail: 54665420@qq.com

Key words: neuregulin-1, ErbB receptors, sepsis, cardiac dysfunction, cardiomyocytes, therapeutic the mechanism of action of NRG-1 intersects with the pathways involved in the pathogenesis of sepsis, it may be applicable as a treatment strategy to numerous pathological processes in sepsis.

\section{Contents}

1. Introduction

2. Presentation of sepsis-induced cardiac dysfunction

3. Effects of NRG-1 on the heart

4. Target of myocardial injury in sepsis and the reversal effect of NRG-1

5. Multiple downstream signaling pathway

6. Current treatment of sepsis

7. NRG-1 treatment and clinical studies

8. Conclusions

\section{Introduction}

Cardiac injury and cardiac insufficiency can be as high as $40-50 \%$ in patients with sepsis (1). In case of impaired cardiac function, the mortality rate can increase from 20 to $70 \%$, and it continues to increase in a number of countries (2). Studies have shown that early improvement of cardiac function can be achieved to significantly improve patient survival rates $(1,2)$. The mechanism of cardiac insufficiency in sepsis is complex. Infection caused in the body not only directly causes myocardial cell damage, but also causes cardiac dysfunction, including myocardial cell structure and function impairment, deregulation of endothelial function and coronary microcirculation, attenuation and activation of myocardial inhibitors and autonomic dysfunction (3). Previously, clinicians have used new drugs such as anti-inflammatory cytokines to treat sepsis cardiac dysfunction, but due to of their relatively singular therapeutic targets, it has not achieved the expected results in clinical applications.

Neuregulin-1 (NRG-1) belongs to the family of epidermal growth factor-like proteins, encoded by 4 neuregulin genes. It is primarily distributed in the nervous and cardiovascular systems. NRG-1 signaling is transduced by the ErbB family, which belongs to the receptor protein tyrosine kinase family, 
and phosphorylation and dimerization of these receptors leads to activation of downstream pathways (4). NRG-1 acts on multiple targets such as heart, blood vessels, nerves and interstitial fluid, and protects the myocardium, promoting the improvement of cardiac function through a combined multifaceted effect. It may have potential and important clinical significance for the treatment and prognosis of sepsis (4-7).

\section{Presentation of sepsis-induced cardiac dysfunction}

Sepsis-3 defines sepsis as a 'life-threatening organ dysfunction caused by abnormal reactions to infection', and recommends sequential organ failure assessment (SOFA) and quick SOFA scores as the basis for diagnosis of sepsis (5-7); however, it does not include the criteria for measuring target organ dysfunction (4-7). Using this definition, 'host response' and 'organ dysfunction' are the two core elements considered to be involved in the complex pathophysiological reactions produced by the body in response to infection (4-7). Current understanding of the complex pathophysiological mechanisms involved in immune and inflammatory responses to sepsis remains incomplete, but it is clear that sepsis involves the immune system, accompanied by inflammation and microcirculatory failure. Despite the improvement of current treatment strategies, and the fact that new treatment concepts and methods are being updated continuously, sepsis and septic shock remain the primary causes of mortality in severe cases (8). A total of $>70 \%$ of sepsis-associated mortalities are due to organ failure, and $50 \%$ are confirmed to exhibit myocardial dysfunction (8). The heart is one of the important target organs in sepsis; toxins, cytokines and complement immune response, oxidative stress and calcium overload, apoptosis and autophagy, as well as energy metabolism disorders, are involved in the occurrence of septic cardiomyopathy (9).

Sepsis-3 included stricter requirements for the diagnosis of sepsis myocardial injury and cardiac insufficiency (5-7). The pathophysiological mechanism of septic heart dysfunction and the effect of NRG-1 on cardiac function in patients with sepsis are as follows: Organ dysfunction is the primary manifestation during sepsis. In the early stages of sepsis, echocardiography measurements demonstrate left ventricular ejection fraction (LVEF) values of $>55 \%$, which may be due to transient myocardial contraction enhancement caused by early increase of adrenaline secretion due to increased vascular permeability, decreased vascular tension and lower stroke output (10). However, this increased compensatory heart rate is insufficient to maintain effective cardiac output. Clinical symptoms are usually accompanied by elevated blood lactate level and decreased oxygen saturation.

With the progression of sepsis, the LVEF gradually decreases, and in addition, the contractile and diastolic functions of the heart gradually decrease, which is accompanied by hypotension, heart failure, arrhythmia and shock (10). In addition to the direct damage caused by inflammatory factors to cardiomyocytes, the dysfunction of mitochondria in cardiomyocytes, dysregulation of the autonomic nervous function, disruption of the myocardial cell calcium cycle, apoptosis, microcirculatory insufficiency, and dysregulation of other processes, leading to cardiac insufficiency, also occur (10). Clinically, it is usually accompanied by increased levels of myocardial enzymatic markers and myocardial necrosis markers, changes in electrocardiogram outputs and hemodynamic disorders (10).

\section{Effects of NRG-1 on the heart}

In the cardiovascular system, NRG-1 is a protein synthesized and released by endothelial cells located in the microvasculature of the heart, which is regulated by neurohumoral factors $(11,12)$. Its receptors include ErbB2 and ErbB4, distributed in atrial and ventricular myocytes, and ErbB3, distributed in the endocardial cushion (12). In recent years, the important role of NRG-1/ErbBs in embryonic development and functional protection of the cardiovascular system has been gradually identified. It has need demonstrated that the activation of NRG-1/ErbBs may activate $\mathrm{PI} 3 \mathrm{~K} / \mathrm{Akt}$, mitogen-activated protein kinase kinase (MEK)/ extracellular signal-regulated kinase (ERK), steroid receptor coactivator (Src)/focal adhesion kinase (FAK), JAK/STAT, and other downstream signaling pathways. In addition, it serves a protective role in myocardial cell structure, promotes myocardial cell proliferation, inhibits apoptosis, regulates mitochondrial function and intracellular calcium circulation, and maintains cardiac microvascular endothelial functions (12). In addition, it may promote angiogenesis and regulate the balance of cardiac autonomic nervous function.

It was identified that NRG-1/ErbBs were activated in the early stage of a heart failure model, which may be associated with the stimulation of NRG-1 secretion by cardiac microvascular endothelial cells by mechanical tension of the ventricular wall. The changes of NRG-1/ErbBs activity are closely associated with the degree of cardiac insufficiency in sepsis $(11,12)$. The dynamic changes of NRG-1/ErbBs may persist in the early stage of myocardial injury and cardiac insufficiency in sepsis, and may become a biological index for evaluating cardiac function in sepsis.

NRG-1/ErbBs were identified to serve an indispensable role in embryonic cardiac development by knocking out NRG-1, ErbB2 and ErbB4 genes $(11,12)$. It has been demonstrated that NRG-1/ErbBs are also indispensable in the mature cardiovascular system $(11,12)$. At present, there are few reports concerning NRG-1/ErbBs and sepsis; however, the biological effects of NRG-1/ErbBs may antagonize a number of complex mechanisms of septic myocardial injury and cardiac insufficiency $(11,12)$, at multiple targets and multiple levels. Therapeutic measures targeting NRG-1/ErbBs will become an effective intervention strategy to reverse septic cardiac insufficiency. Intervention using NRG-1 in sepsis may serve a comprehensive role in protecting the structure and function of cardiomyocytes and subcellular processes, inhibiting endothelial cell injury, downregulating myocardial inhibitors, and regulating cardiac autonomic nervous function, by activating the direct and indirect cardiovascular protection of NRG-1/ErbBs. In addition, NRG-1 may serve a key role in the maturation of the cardiac plexus, and if such effects are attenuated or affected, it may lead to a decrease in cardiomyocyte maturity, function and survival (12).

A number of biological effects mediated by NRG-1/ErbBs have been demonstrated to antagonize the mechanisms of cardiac insufficiency in sepsis at multiple target sites. Therefore, in view of the mechanism of cardiac insufficiency caused by sepsis myocardial injury, exploring endogenous bioactive 
substances with multi-target regulation and protective effects on heart and blood vessels may result in the identification of potential diagnostic markers and intervention targets for the diagnosis and treatment of sepsis.

\section{Target of myocardial injury in sepsis and the reversal effect of NRG-1}

As the pathogenesis of septic cardiomyopathy and the mechanism of action of NRG-1/ErbBs on cardiac function have numerous intersecting facets, the present review has summarized the primary role and development of NRG-1/ErbBs in cardiac dysfunction in sepsis.

When sepsis occurs, the mechanism of cardiomyocytes dysfunction is complex, and may include apoptosis of cardiomyocytes, myofibrillar lysis, mitochondrial dysfunction and intracellular calcium regulation disorders $(9,13)$. NRG-1/ErbBs participate in the proliferation and development of cardiomyocytes, promote the structural integrity and orderly arrangement of cardiomyocytes, and have a direct protective effect on cardiomyocytes (14). Previous study has examined the effect of NRG-1 on cardiac structure and function in sepsis rats; cecum ligation and perforation (CLP) was used to establish a sepsis rat model (15). It was identified that intravenous administration of recombinant human NRG-1 (rhNRG-1) significantly improved cardiac function and myocardial cell pathology in rats $24 \mathrm{~h}$ after CLP (15). This study also demonstrated that NRG-1/ErbBs have important protective effects on the structure and function of cardiomyocytes and their organelles (15).

Mitochondrial function of cardiomyocytes. Myocardial mitochondrial matrix edema accompanied by cystic changes of mitochondrial ridge was observed in patients with sepsis (16). Animal models also demonstrated that sepsis resulted in the opening of mitochondrial membrane permeability transition pores (mPTP), destruction of membrane potential, abnormal activity of cytochrome oxidase, increase of nitric oxide (NO) and reactive oxygen species (ROS) in the mitochondria, depletion of ATP, swelling and apoptosis of the mitochondria, and promotion of the dysfunction of myocardial cells (17). It was identified that NRG-1 isozyme intervention improved the mitochondrial function of myocardial cells in rats with myocardial infarction (18). NRG-1 was demonstrated to increase the mitochondrial membrane potential, inhibit the release of cytochrome $\mathrm{C}$, enhance the activity of ATPase, improve respiratory function, and decrease the dysfunction of myocardial cells (19). Therefore, NRG-1 not only protects the function of the mitochondria, but also serves an important role in the protection of the endoplasmic reticulum. In 2016, it was identified that intravenous administration of NRG-1 significantly decreased endoplasmic reticulum stress and myocardial infarction area (20), thereby protecting myocardial cells in rat model of myocardial ischemia/reperfusion $(19,20)$.

Apoptosis of cardiomyocytes. During sepsis, the Akt pathway and its downstream signaling factors are activated, and the expression levels of the apoptotic factors Bax/Bcl-2, caspase-3 and platelet-derived growth factor (PDGF) are increased. Together, these changes result in vascular endothelial cell damage and cardiomyocyte apoptosis (18-20).
Previous study has suggested that the activation of endogenous NRG-1/ErbBs may decrease myocardial apoptosis in rats with myocardial infarction (20). In a previous study, NRG-1 was combined with degradable PLGA microspheres and injected into the periphery of myocardial infarction areas. It was identified that NRG-1 promoted the expression levels of $\mathrm{mF} 2 \mathrm{c}^{+}$/antigen $\mathrm{Ki}-67^{+}$in cardiac cells, which are markers of myocardial cell proliferation, and ultimately decreased infarct size and improved cardiac function (21). In addition, a change in cytochrome $\mathrm{C}$ can directly affect the oxidative phosphorylation of the mitochondria, release apoptotic factors into the cytoplasm, and participate in cardiomyocyte apoptosis. NRG-1 is able to inhibit the release of cytochrome $\mathrm{C}$, thereby decreasing the level of dysfunction of cardiomyocytes (18). In addition, in 2014, it was identified that NRG-1 promoted mitosis of myocardial cells, decreased the level of apoptosis, and enhanced cardiac function by injecting NRG-1 into the periphery of the infarction area using hydrogel (22).

Calcium circulation disorder is the direct cause of cardiac function decline due to sepsis-associated myocardial injury (23). Transient decreases in calcium concentration, and decreases in L-type calcium current density in the myocardial cell membrane, the expression levels of alanine receptors and the sarcoplasmic reticulum calcium pump, functioning of phosphoproteins, in sarcoplasmic reticulum uptake, and in the function of the sodium-calcium exchanger all contribute to disruption of calcium homeostasis (24). Through the addition of NRG-1 into cultured rat cardiac myocytes, a previous study confirmed that NRG-1/ErbBs participated in the regulation of calcium homeostasis in cardiac myocytes, by increasing the recovery of sarcoplasmic reticulum calcium, calcium transients, and upregulating the expression of the sarcoplasmic reticulum calcium pump and phosphoproteins (24). In vivo studies have demonstrated that activation of NRG-1/ErbBs can improve cardiac function in model animals, and the associated cellular and subcellular protective mechanisms may serve a preventive and therapeutic role in cardiac insufficiency caused by septic myocardial injury (23-25). It was observed that the structural and functional changes of cardiac myocytes and subcellular processes in sepsis directly caused the decline of cardiac contractile function $(15,23,24)$, which is an important target for the prevention and treatment of cardiac insufficiency in sepsis.

Function of microvascular endothelial cells. Microcirculation is first involved in sepsis. Cytokines and inflammatory mediators released by systemic inflammatory reaction may lead to injury of vascular endothelial cells, activation of leukocytes and platelets, and further, to the release of inflammatory and adhesion factors, dysfunction of the coagulation system and microthrombosis in capillaries, finally resulting in multiple organ failure (25). The decline of cardiac and systemic microvascular endothelial cell function eventually leads to a worsening of cardiac insufficiency in sepsis.

The expression levels of vascular cell adhesion factor (VCAM-1), intercellular adhesion molecule (ICAM), E-selectin, and von Willebrand factor (vWF) increase in patients with sepsis, which results in local neutrophil infiltration in the heart. Concomitantly, cardiac microvascular endothelial cells also suffer from swelling and necrosis and deposition of fibrin in the blood vessels, resulting in increased resistance to coronary microcirculation and uneven 
distribution of blood flow, aggravating myocardial ischemic damage $(7,26)$. A previous study identified that NRG-1 may prevent endothelial hyper permeability, decrease the expression of VCAM-1 and E-selectin in microvascular endothelial cells, and decrease the adhesion of neutral cells to endothelial cells, thereby alleviating endothelial injury (27).

Studies have identified that the integrity of the vascular endothelial structure and its function in patients with sepsis directly affects disease progression (28). The involvement of microcirculation in the whole body results in decreased vascular responsiveness, microcirculation disturbance in vital organs, and an imbalance of inflammatory cell regulation (28). Endocardial and vascular endothelial cells of the heart synthesize and release NRG-1, which is critical for the development of the adult circulatory system and maintenance of cardiovascular function (28-30). Recently, an increasing number of studies have demonstrated that NRG-1 is a regulator of vascular endothelial regeneration. Previous studies have identified that NRG-1/ErbBs may promote the proliferation of microvascular endothelial cells and decrease apoptosis, while serving an important role in maintaining endothelial function and promoting angiogenesis (28-33). Parodi and Kuhn (29), demonstrated that NRG-1 and ErbB receptors are expressed in vascularendothelial cells, and that the stimulation of endothelial cells in vitro may induce the formation of vascular endothelial cells. The results from the study by Hedhli et al (30), indicated that angiogenesis and arteriogenesis were induced following ligation of the femoral artery, and that NRG-1 was a crucial factor in this process. In addition, the injection of exogenous NRG-1 promoted this process.

It has been suggested that the activation of NRG-1/ErbBs can transcriptionally activate proteins by phosphorylation, and then stimulate the secretion of vascular endothelial cells by paracrine action to produce an endothelial regeneration effect (31). Local NRG-1 intervention in the ischemic myocardium can induce endothelial progenitor cell recruitment (31). In addition, it increased the density of $\alpha$-smooth muscle actin ${ }^{+}$ and caveolin- $1^{+}$cells, which are markers of angiogenesis and microangiogenesis (21). The effects of NRG-1 treatment on myocardial angiogenesis and signal transduction of vascular endothelial growth factor/vascular endothelial growth factor receptor 2 and angiopoietin-1 (Ang-1)/angiopoietin-1 receptor in diabetic cardiomyopathy rats were studied using animal models. The results indicated that NRG-1 treatment significantly increased the expression of vascular endothelial growth factor (VEGF) and Ang-1 in coronary artery smooth muscle cells (32). The intervention of NRG-1 may increase myocardial angiogenesis through the direct effect of NRG-1 and increased expression of VEGF and Ang-1 (30). NRG-1 is a regulator of angiogenesis, and previous data has indicated that VEGF and Ang-1 regulate myocardial angiogenesis and growth through the NRG-1/ErbBs signaling pathway $(33,34)$. These results may help to identify a novel method of reversing the damage incurred in the vascular regeneration processes in coronary artery disease.

Therefore, activation of NRG-1/ErbBs can inhibit microvascular endothelial injury in sepsis. By evaluating the function of microvascular endothelial cells and applying potential drugs to improve endothelial function, the diagnosis and treatment sepsis may be improved.
Expression of myocardial depression factors. Inflammatory mediators activated during sepsis are currently considered one of the important causes of cardiac insufficiency in sepsis (13,34-42). These include tumor necrosis factor- $\alpha$ (TNF- $\alpha)$, interleukin (IL)-1, IL-2, IL-4, IL-6, IL-8, IL-10, and interferon- $\gamma$ (IFN- $\gamma$ ), which are also known as myocardial depressant factors (34). There is evidence to show that NRG-1/ErbBs can regulate neurological and humoral immunity and exert anti-inflammatory and anti-oxidative effects. In vivo and in vitro experiments have identified that NRG-1 mutation may cause immune system dysfunction and increase the expression of TNF- $\alpha$, IL- 6 , and IL-8 in lymphoblasts (34).

With increased blood circulation, myocardial inhibitors may directly or indirectly damage the myocardium and affect cardiac contractile function (33-35). The inhibitory effects of TNF- $\alpha$, IL- 1 and IL- 6 can inhibit myocardial contractile force and decrease LVEF. Their direct negative inotropic effects are mediated by NO synthase. Pathan et al (36) identified that the level of IL-6 in patients with sepsis was directly associated with cardiac insufficiency. In addition, another study also confirmed the role of IL- 6 in the pathogenesis of organ dysfunction in children with sepsis $(36,37)$. It can cause cardiac dysfunction by activating the p38 mitogen-activated protein kinase pathway (36). In the CLP model of septic shock in mice, the severity of sepsis could be predicted by IL-6 levels, $6 \mathrm{~h}$ after the onset of sepsis (38).

NRG-1 may decrease the levels of TNF- $\alpha$, IL-1 $\beta$ and IL-6 in the blood circulation of septic rats, and decrease the level of macrophage migration inhibitory factor (MIF) in myocardial tissue (13). NRG-1 binds to ErbB, which initiates intracellular signal transduction and the development of a series of cellular responses, including cell proliferation and survival $(39,40)$. Mencel et al (41) studied the effects of NRG-1 on the synthesis of TNF- $\alpha$ and IL- 6 , which are the key mediators of inflammation. Secretory TNF- $\alpha$ and IL- 6 were collected from cultured cells, and the results demonstrated that NRG-1 decreased the secretion levels of TNF- $\alpha$ and IL- 6 by 33 and $80 \%$, respectively. The NRG-1 signaling pathway may antagonize the inflammatory response of microglia, decrease the inflammatory response and alleviate lipopolysaccharide (LPS)-induced TNF- $\alpha$ synthesis in microglia.

A previous study also identified that NRG-1 may alleviate delayed neuronal injury induced by neurotoxic drugs by decreasing the expression of IL- 6 and IL- $1 \beta$ in experimental studies of other diseases (41). A previous study has indicated that NRG-1 may decrease the levels of circulatory and local pro-inflammatory factors like TNF- $\alpha$, IL- 6 and IL-1 $\beta$, and significantly improve the survival rate of experimental cerebral malaria rats (42). Therefore, the inhibitory effects of NRG-1/ErbBs on inflammatory factors, locally or in the bloodstream, appear to attenuate the adverse effects of certain myocardial inhibitors on cardiac function in sepsis.

Autonomic nervous system homeostasis. Localized processes associated with autonomic nervous system dysfunction often occur in sepsis. These include apoptosis of glial cells and neurons in the cardiovascular center, imbalance of sympathetic and vagal nerve function, abnormal secretion of neurohormones, and low reactivity of parts of the heart, such as the sinoatrial node to autonomic nerve regulation, all of which 
may affect cardiovascular activity and aggravate cardiac insufficiency (43).

The NRG-1/ErbBs pathway is particularly important in early cardiac and neurological repair processes. A previous experimental study suggested that insufficient expression of NRG-1 in the process of establishing a cardiac plexus may result in decreased cardiac maturation, function and survival (44).

Previous studies have suggested that drug activation of the peripheral and central nervous pathways may inhibit inflammation, thereby achieving an equivalent effect to that observed in the treatment of sepsis $(12,45)$. Cardiac autonomic nervous dysfunction is commonly observed in patients with sepsis, and autonomic nervous dysfunction may aggravate cardiac insufficiency. Previous studies have identified that NRG-1/ErbBs also serve a role in the regulation of the cardiovascular center. Intrathecal injection of NRG-1 inhibited sympathetic hyperexcitability and improved heart failure caused by pressure overload $(46,47)$.

The results from Matsukawa et al (47) demonstrated a reversible decrease in heart rate variability (HRV) indicators, reflecting cardiac autonomic nervous function, in healthy volunteers following intravenous endotoxin injection. Follow-up studies also confirmed that the decrease of HRV in patients with sepsis was a marker of poor prognosis. NRG-1/ErbBs are involved in the regulation of cardiac autonomic nervous homeostasis (47). The secretion of NRG-1 is regulated by neurohormones. Other studies have identified that adrenaline and angiotensin II can change the expression of NRG-1 in myocardial microvascular endothelial cells $(33,34)$. NRG-1 may prevent Ang II-induced left ventricular concentric remodeling and diastolic dysfunction, without affecting arterial blood pressure $(33,34)$. These effects help explain the beneficial effects of NRG-1 in heart failure. Furthermore, these results suggest that NRG-1/ErbBs are involved in the regulation of autonomic nervous homeostasis in the cardiovascular system.

In addition, according to the central mechanism of autonomic nervous imbalance in sepsis, in vivo and in vitro experiments have confirmed that NRG-1 may promote the growth and differentiation of glial cells, inhibit neuronal apoptosis, and have a neuroprotective effect (48). This non-cardiovascular biological effect may also serve a role in antagonizing the imbalance of the autonomic nervous system in sepsis.

\section{Multiple downstream signaling pathway}

It has been confirmed that NRG-1 improves cardiac function by regulating multiple downstream signaling pathways, including mitogen-activated protein kinase (MAPK)/ERK, PI3K/Akt, transforming protein RhoA (RhoA)/Rho-associated protein kinase (ROCK), Janus kinase 2 (JAK2)/signal transducer and activator of transcription 3 (STAT3), and Src/FAK (48-77). The effect of NRG-1/ErbBs on the regulation of sarcomeric actin polymerization in cardiomyocytes may be antagonized by specific inhibitors of PI3K and ERK (48). Jabbour et al (49) added NRG-1 to the perfusate of isolated functional heart cells and observed that it protected cardiomyocytes, improved cardiac function, and was associated with the increased phosphorylation of Akt, ERK and STAT3. NRG-1/ErbB signaling serves a critical role in cardiac repair and regeneration in heart failure (49). A previous study suggested that activation of the NRG-1/ErbBs system may be one of the underlying mechanisms of cardiac repair and regeneration after myocardial infarction (49). Activating the signal transduction of ErbB2, ErbB4 and PI3K/Akt by upregulating NRG-1 expression may help to promote cardiomyocyte repair.

A previous study indicated that the myocardial inhibition of IL-6, an inflammatory mediator activated during sepsis, depends on p38 MAPK signaling pathway (50), which regulates inflammation, cell proliferation, apoptosis, differentiation and other processes $(47,48)$. By inhibiting the p38 MAPK pathway, the effect of reversing the negative muscle strength of IL- 6 can be achieved $(46,47)$. Experimental studies have confirmed the activation of p38 MAPK in myocardium, lung, and brain tissue during sepsis $(12,43,45,48)$, suggesting that MAPK has a broad role in the pathophysiology of multiple organ dysfunction in sepsis. In an animal model of LPS-induced acute lung injury, it was identified that the downregulation of the downstream NF- $x$ B pathway alleviated lung injury by inhibiting the p38 MAPK signaling pathway (51). In another animal models, the p38 MAPK pathway was also demonstrated to be involved in the disease progression of sepsis; Zhou et al (52) identified that MAPK pathways exhibited significant levels of activation in children with septic shock.

The inhibitory effects of TNF- $\alpha$ and endotoxins on the heart muscle are also transduced through the MAPK pathway (53). During myocardial ischemia-reperfusion, MAPK activation induces cardiomyocyte apoptosis, increases adhesion molecule expression and decreases myocardial myofilament sensitivity (54). In addition, the inhibition of MAPK decreased myocardial TNF- $\alpha$ expression and improved cardiac function and survival in endotoxemic rats (53). The results from RNA expression studies in patients with sepsis have demonstrated an increased transcription levels of MAPK upstream activator genes (53). The increased expression of the IL-6 receptor, JAK2 and Akt, and downstream mediators including transcription factors such as NF- $\chi \mathrm{B}$ and activating transcription factors, MAPK-activated protein kinases and inflammatory cytokines, confirms the important role of the MAPK pathway in sepsis (55). Other inflammatory mediators with myocardial inhibitory effects such as the complement component C5a and the pro-inflammatory cytokine TNF- $\alpha$, have also been shown to activate the p38 MAPK pathway $(32,55)$.

In addition, previous studies have identified that in other forms of systemic inflammatory activation, such as burns (56), ischemia-reperfusion (57), and cardiopulmonary shunts $(58,59)$, MAPK activation is associated with multiple organ dysfunction. Therefore, these data have improved the current understanding of the association between NRG-1 and the MAPK signaling pathway. Upstream or downstream activators and inhibitors that regulate this pathway have garnered increasing attention in studies of animal and human sepsis models (60-63).

NRG-1 is produced by endocardial and myocardial microvascular endothelial cells and binds to the receptor tyrosine kinase of the ErbB family on the cell surface of adjacent cardiomyocytes, activating the PI3K/Akt signaling pathway (64). The PI3K/Akt pathway has been demonstrated to serve a key cardioprotective role in myocardial ischemia/reperfusion $(65,66)$. PI3K/Akt signaling is the central regulator of the 


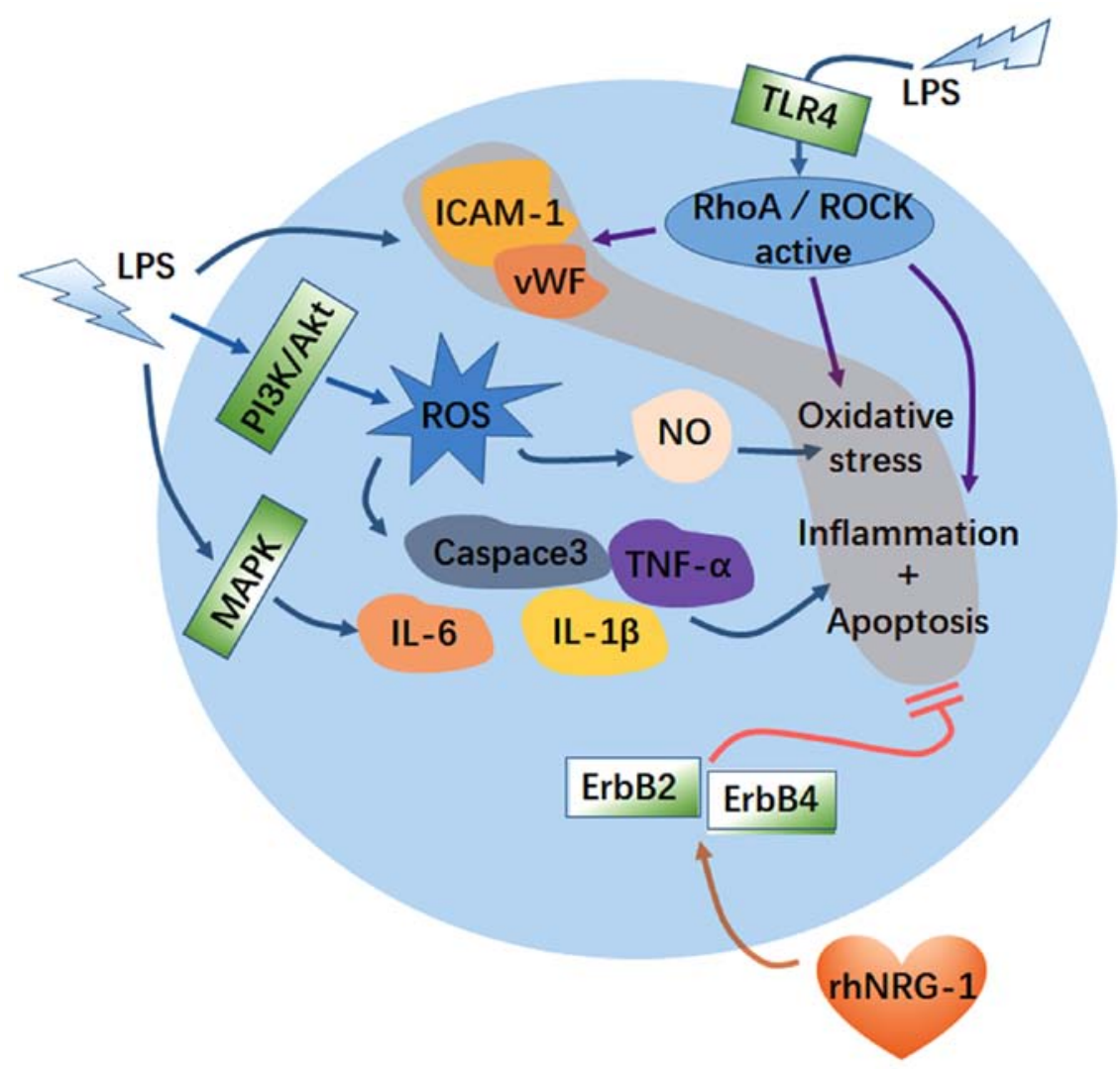

Figure 1. Exogenous LPS directly leads to an increase in the production of white adhesion molecules and other cytokines such as ICAM-1 and vWF. In addition, LPS activates MAPK, PI3K/Akt and other downstream signaling pathways, producing IL-6, IL-1 $\beta$, TNF- $\alpha$ and other inflammatory factors, and excessive oxidative stress leads to a significant amount of NO production. Activation of the RhoA/ROCK signaling pathway produces the same effect. Conversely, NRG-1/ErbBs may attenuate or inhibit these processes. It serves a protective role in myocardial cell structure, inhibits apoptosis and oxidative stress and decreases inflammation. LPS, lipopolysaccharide; ICAM-1, intercellular adhesion molecule 1; vWF, von Willebrand factor; MAPK, mitogen-activated protein kinase; IL, interleukin; TNF- $\alpha$, tumor necrosis factor; NO, nitric oxide; NRG-1, neuregulin-1; rhNRG-1, recombinant human NRG-1; ROS, reactive oxygen species; TLR4, toll-like receptor 4; rhNRG-1, recombinant human NRG-1; RhoA/ROCK, transforming protein Rho/Rho-associated protein kinase.

NRG/ErbB signaling network (67). It was also identified that activation of PI3K/Akt signaling pathway may alleviate the inflammatory response induced by LPS, and improve cardiac dysfunction in sepsis $(68,69)$. NRG-1 inhibits cardiomyocyte apoptosis caused by sepsis cardiac dysfunction, and previous studies have indicated that a number of cardioprotective agents act by inhibiting the opening of the mPTP via the PI3K/Akt signal-dependent pathway $(68,69)$. rhNRG-1 has significant potential for use as a therapeutic agent for apoptosis, induced by oxidative stress. Wang et al (17) suggested that NRG-1 may decrease myocardial infarct size and apoptosis in vivo. The PI3K inhibitor LY294002 suppressed the phosphorylation of AKT and abolished the cardioprotective effects induced by NRG-1. These results indicate the therapeutic potential of NRG-1 as a pharmacological postconditioning agent against myocardial reperfusion injury (17).

Previous studies have identified that endotoxins activate the RhoA/Rho kinase (RhoA/ROCK) signaling pathway, which induces mitochondrial autophagy by altering the cytoskeleton, causing cardiac dysfunction in mice $(53,54)$. Activation of the RhoA/ROCK signaling pathway decreases NO availability, upregulates the expression of the leukocyte adhesion factor ICAM-1, and participates in various inflammatory responses. In addition, RhoA/ROCK signaling causes endothelial cell shedding, increases serum levels of vWF, and increases the permeability of the vascular endothelium. RhoA activation has been demonstrated to be involved in oxidative stress (70). ROCK regulates the development of cardiovascular diseases by inducing the expression of NO synthase in endothelial cells (71). Insufficient NO production in vascular endothelial cells leads to impaired endothelium-dependent vasodilation (72). In addition, inhibition of the RhoA/ROCK pathway activates NO production and attenuates vascular inflammation (73).

NRG-1 has also been demonstrated to inhibit RhoA activation (Fig. 1), decrease myosin light chain phosphorylation, and decrease microvascular endothelial cell permeability, which in turn inhibits the microcirculatory inflammatory response (54). NRG-1-induced tumor cell motility is known to negatively regulate ROCK signaling (74). A previous study demonstrated that activation of RhoA/Rho kinase signaling promotes the level of VEGF in vitro and induces endothelial cell migration, angiogenesis and cytoskeletal changes; by contrast, the inhibition of Rho kinase prevented mechanical wounding (75). The RhoA/ROCK pathway induces thrombin-induced ICAM-1 expression via the activation of I B kinase (76) and exacerbates LPS-induced endothelial hyper-permeability by mediating the formation of stress fibers in endothelial cells. Therefore, inhibition of RhoA/ROCK activation may improve vascular hyper-permeability in sepsis (77). 
Although the associated signaling pathways are less-studied in sepsis, it has been suggested that MEK/ERK and JAK2/STAT3 may be used as therapeutic targets for the protection of organs during sepsis (51). Therefore, the regulatory effect of NRG-1 on multiple signaling pathways may be important in antagonizing myocardial injury in sepsis.

\section{Current treatment of sepsis}

At present, there is no specific treatment for septic myocardial injury and cardiac insufficiency. Available treatment options primarily include early anti-infective drugs, fluid resuscitation and continuous blood purification. In addition, decreasing of myocardial interstitial edema, organ function support, positive inotropic drugs and drugs for improving myocardial microcirculation are occasionally administered.

At present, levosimendan, angiotensin and endothelin receptor antagonists are the primary drugs used to improve cardiac function during sepsis. Levosimendan is a calcium sensitizer that enhances myocardial contractility. However, a small-scale study failed to demonstrate that levosimendan could significantly decrease the mortality rate of patients with sepsis (78). Endothelin receptor antagonists may improve cardiac function by blocking endothelin receptors A and B. Bonner et al (79) identified that sepsis-induced acute heart failure was attributed to the activation of the endothelin system. It has been demonstrated that endothelin receptor antagonists have therapeutic significance: A previous study indicated that decreases in the circulatory levels of endothelial damage markers was observed in patients treated with nangibotide, a Triggering receptor expressed on myeloid cells-1 pathway inhibitor (80).

Positive inotropic drugs increase myocardial cell calcium concentration, thereby increasing myocardial oxygen consumption, and have a poor therapeutic effect on septic heart dysfunction (15,78-80). In addition, the treatment is expensive and the drugs are single-target, therefore, it is important to identify a novel drug that acts on multiple targets.

\section{NRG-1 treatment and clinical studies}

Our previous study demonstrated that NRG-1 improved cardiac function and protects cardiac myocytes in CLP-induced septic rats by inhibiting immune inflammation, release of myocardial inhibitors and over activation of the renin-angiotensin-aldosterone system (15). The results of the study by Fang et al (81) indicated that pretreatment with NRG-1 analogues may alleviate myocardial ischemia-reperfusion injury, and significantly improve myocardial function impairment. The downregulation of NRG-1/ErbB4 signaling pathway is closely associated with myocardial dysfunction in septic rats $(15,81)$. NRG-1 preconditioning may attenuate myocardial function impairment in septic rats, and ErbB4 antagonists can inhibit this effect $(15,81)$.

Other studies have demonstrated that NRG-1 may improve symptoms in animal models of heart disease including ischemia, dilated myocardial function, and viral cardiomyopathy $(82,83)$. NRG-1 promotes cardiomyocyte growth and survival (84), regulates myofibrillar disorders (85), maintains mitochondrial function and integrity (86), stimulates glucose uptake (87), and increases sarcoplasmic reticulum calcium uptake (88). These effects of NRG-1 are beneficial for cardiac function.

In addition, NRG-1/ErbBs also exert systemic effects, such as an inhibition of the inflammatory response, mitigation of oxidative stress, protection of central and peripheral nerve functions, and extensive protection in a variety of pathological conditions (89-91). Increasing evidence suggests that NRG-1 intervention is a promising treatment for heart failure (14,89-91). In a clinical study involving 899 patients with heart failure, elevated levels of NRG-1 in the blood were positively correlated with the severity of heart failure (89-92). In addition, high levels of NRG-1 were independently associated with mortality and heart transplantation, which may be exploited as a new biomarker for heart failure diagnosis and prognosis evaluation (26).

A phase II clinical trial (trial no. ACTRN12607000330448) of rhNRG-1 short-term continuous (10 or 11 days) intravenous administration for heart failure was conducted. The results demonstrated that rhNRG-1 may increase cardiac output, ejection fraction, and decrease pulmonary wedge pressure. Peripheral vascular resistance and improved cardiac function was observed within $2 \mathrm{~h}$ of rhNRG-1 intravenous infusion, and the effect lasted until the 12th week of the study follow-up (91). In addition, patients tolerated the treatment well, without adverse reactions (92). All of these data suggest that NRG-1 intervention may be a useful therapeutic strategy for improving cardiac function, and exhibits great prospects for clinical application.

\section{Conclusions}

In summary, sepsis is an uncontrolled inflammatory reaction that may result in multiple organ dysfunction. The pathogenesis of sepsis involves multiple factors, which together can lead to patient mortality. At present, clinical treatment for sepsis to improve symptoms is poor, and a multi-target drug is required. NRG-1 may serve an important role in the treatment of septic myocardial injury by targeting the multiple pathogenesis of sepsis, thereby providing new treatment options for patients with sepsis and improving disease prognosis.

The NRG-1/ErbBs system exerts a multifaceted network effect on cardiovascular protection. Myocardial injury cardiac dysfunction is a common complication of sepsis, driven by a complex mechanism; early assessment and prompt intervention are the key to decreasing mortality. NRG-1 may specifically antagonize the associated mechanisms of myocardial depression in sepsis, thereby exerting a more comprehensive therapeutic effect. The activation and changes of NRG-1/ErbBs system may be associated with the course of cardiac dysfunction in sepsis myocardial injury. Monitoring its activity may provide a new biomarker for evaluating cardiac function in sepsis; early NRG-1 intervention may serve a role in directly protecting the structure and function of cardiomyocytes, and improve cardiac dysfunction in sepsis myocardial injury by promoting endothelial function, antagonizing myocardial inhibitory factor, and regulating cardiac autonomic balance.

The presence of different types of NRGs indicates the complexity of the NRG-1/ErBbs signaling system, and the numerous effects of NRG-1 on the cardiovascular system is 
promising for the treatment of sepsis, and it may gradually be adopted into clinical practice. Further study of the association between NRG-1/ErbBs and sepsis may provide a new direction for the diagnosis of sepsis cardiac dysfunction to identify potential diagnostic markers and intervention targets. NRG-1 has the potential to be used as a therapeutic tool in clinical diagnosis and treatment of sepsis, and its application will help to improve the management of cardiac complications in sepsis in the future.

\section{Acknowledgements}

Not applicable.

\section{Funding}

The present study was supported by the National Natural Science Foundation of China (grant no. 81772044), Science Foundation of Wuhan Health and Family Planning Commission (grant no. WX17B09), Natural Science Foundation of Hubei Province (grant no. 2017CFC824), Wuhan University Young Teachers Project (grant no. 2042018kf0199).

\section{Availability of data and materials}

Not applicable.

\section{Authors' contributions}

WK wrote the manuscript and wrote the introduction, 'Presentation of sepsis-induced cardiac dysfunction', 'Effects of NRG-1 on the heart' and concluding sections. YC wrote the 'Target of myocardial injury in sepsis and the reversal effect of NRG-1' sections. XW and LCZ scientifically edited the manuscript. FZ wrote the 'Multiple downstream signaling pathway' sections. LZ wrote the 'Current treatment of sepsis' sections. LW wrote the abstract. All authors read and approved the final manuscript.

\section{Ethics approval and consent to participate}

Not applicable.

\section{Patient consent for publication}

Not applicable.

\section{Competing interests}

The authors declare that they have no competing interests.

\section{References}

1. Kuang L, Zhu Y, Zhang J, Wu Y, Tian K, Chen X, Xue M, Tzang FC, Lau B, Wong BL, et al: A novel cross-linked haemoglobin-based oxygen carrier is beneficial to sepsis in rats. Artif Cells Nanomed Biotechnol 47: 1496-1504, 2019.

2. Moore JX, Donnelly JP, Griffin R, Howard G, Safford MM and Wang HE: Defining sepsis mortality clusters in the united states. Crit Care Med 44: 1380-1387, 2016.

3. Zaky A, Deem S, Bendjelid K and Treggiari MM: Characterization of cardiac dysfunction in sepsis: An ongoing challenge. Shock 41: $12-24,2014$.
4. Salvany S, Casanovas A, Tarabal O, Piedrafita L, Hernández S, Santafé M, Soto-Bernardini MC, Calderó J, Schwab MH and Esquerda JE: Localization and dynamic changes of neuregulin-1 at C-type synaptic boutons in association with motor neuron injury and repair. FASEB J 33: 7833-7851, 2019.

5. Seymour CW, Liu VX, Iwashyna TJ, Brunkhorst FM, Rea TD, Scherag A, Rubenfeld G, Kahn JM, Shankar-Hari M, Singer M, et al: Assessment of clinical criteria for sepsis: For the third international consensus definitions for sepsis and septic shock (Sepsis-3). JAMA 315: 762-774, 2016.

6. Shankar-Hari M, Phillips GS, Levy ML, Seymour CW, Liu VX, Deutschman CS, Angus DC, Rubenfeld GD and Singer M: Sepsis Definitions Task Force: Developing a new definition and assessing new clinical criteria for septic shock: For the third international consensus definitions for sepsis and septic shock (Sepsis-3). JAMA 315: 775-787, 2016.

7. Singer M, Deutschman CS, Seymour CW, Shankar-Hari M, Annane D, Bauer M, Bellomo R, Bernard GR, Chiche JD, Coopersmith CM, et al: The third international consensus definitions for sepsis and septic shock (Sepsis-3). JAMA 315: 801-810, 2016.

8. Potz BA, Sellke FW and Abid MR: Endothelial ROS and impaired myocardial oxygen consumption in sepsis-induced cardiac dysfunction. J Intensive Crit Care 2: 20, 2016.

9. Cimolai MC, Alvarez S, Bode C and Bugger H: Mitochondrial mechanisms in septic cardiomyopathy. Int J Mol Sci 16: 17763-17778, 2015

10. Martin L, Derwall M, Thiemermann C and Schürholz T: Heart in sepsis: Molecular mechanisms, diagnosis and therapy of septic cardiomyopathy. Anaesthesist 66: 479-490, 2017. (In German).

11. D'Uva G, Aharonov A, Lauriola M, Kain D, Yahalom-Ronen Y, Carvalho S, Weisinger K, Bassat E, Rajchman D, Yifa O, et al: ERBB2 triggers mammalian heart regeneration by promoting cardiomyocyte dedifferentiation and proliferation. Nat Cell Biol 17: 627-638, 2015.

12. Brown D, Samsa LA, Ito C, Ma H, Batres K, Arnaout R, Qian L and Liu J: Neuregulin-1 is essential for nerve plexus formation during cardiac maturation. J Cell Mol Med 22: 2007-2017, 2018.

13. Hobai IA, Edgecomb J, LaBarge K and Colucci WS: Dysregulation of intracellular calcium transporters in animal models of sepsis-induced cardiomyopathy. Shock 43: 3-15, 2015.

14. Yutzey KE: Regenerative biology: Neuregulin 1 makes heart muscle. Nature 520: 445-446, 2015.

15. Zhou Q, Pan X, Wang L, Wang X and Xiong D: The protective role of neuregulin-1: A potential therapy for sepsis-induced cardiomyopathy. Eur J Pharmacol 788: 234-240, 2016.

16. Takasu O, Gaut JP, Watanabe E, To K, Fagley RE, Sato B, Jarman S, Efimov IR, Janks DL, Srivastava A, et al: Mechanisms of cardiac and renal dysfunction in patients dying of sepsis. Am J Respir Crit Care Med 187: 509-517, 2013.

17. Wang F, Wang H, Liu X, Yu H, Zuo B, Song Z, Wang N, Huang W and Wang G: Pharmacological postconditioning with Neuregulin-1 mimics the cardioprotective effects of ischaemic postconditioning via ErbB4-dependent activation of reperfusion injury salvage kinase pathway. Mol Med 24: 39, 2018.

18. Galindo CL, Kasasbeh E, Murphy A, Ryzhov S, Lenihan S, Ahmad FA, Williams P, Nunnally A, Adcock J, et al: Anti-remodeling and anti-fibrotic effects of the neuregulin-lbeta glial growth factor 2 in a large animal model of heart failure. J Am Heart Assoc 4: e528, 2015.

19. Fang SJ, Li PY, Wang CM, Xin Y, Lu WW, Zhang XX, Zuo S, Ma CS, Tang CS, Nie SP, et al: Inhibition of endoplasmic reticulum stress by neuregulin-1 protects against myocardial ischemia/reperfusion injury. Peptides 88: 196-207, 2017.

20. Cai MX, Shi XC, Chen T, Tan ZN, Lin QQ, Du SJ and Tian ZJ: Exercise training activates neuregulin 1/ErbB signaling and promotes cardiac repair in a rat myocardial infarction model. Life Sci 149: 1-9, 2016.

21. Formiga FR, Pelacho B, Garbayo E, Imbuluzqueta I, Díaz-Herráez P, Abizanda G, Gavira JJ, Simón-Yarza T, Albiasu E, Tamayo E, et al: Controlled delivery of fibroblast growth factor-1 and neuregulin-1 from biodegradable microparticles promotes cardiac repair in a rat myocardial infarction model through activation of endogenous regeneration. J Control Release 173: 132-139, 2014.

22. Cohen JE, Purcell BP, MacArthur JW Jr, Mu A, Shudo Y, Patel JB, Brusalis CM, Trubelja A, Fairman AS, Edwards BB, et al: A bioengineered hydrogel system enables targeted and sustained intramyocardial delivery of neuregulin, activating the cardiomyocyte cell cycle and enhancing ventricular function in a murine model of ischemic cardiomyopathy. Circ Heart Fail 7: 619-626, 2014. 
23. Sepúlveda M, Gonano LA, Viotti M, Morell M, Blanco P, López Alarcón M, Peroba Ramos I, Bastos Carvalho A, Medei E and Vila Petroff M: Calcium/Calmodulin protein kinase II-dependent ryanodine receptor phosphorylation mediates cardiac contractile dysfunction associated with sepsis. Crit Care Med 45: e399-e408, 2017.

24. Brero A, Ramella R, Fitou A, Dati C, Alloatti G, Gallo MP and Levi R: Neuregulin-1beta1 rapidly modulates nitric oxide synthesis and calcium handling in rat cardiomyocytes. Cardiovasc Res 88: 443-452, 2010.

25. Candel FJ, Borges Sá M, Belda S, Bou G, Del Pozo JL, Estrada O, Ferrer R, González Del Castillo J, Julián-Jiménez A, Martín-Loeches I, et al: Current aspects in sepsis approach. Turning things around. Rev Esp Quimioter 31: 298-315, 2018.

26. Bermejo-Martin JF, Martín-Fernandez M, López-Mestanza C, Duque $\mathrm{P}$ and Almansa R: Shared features of endothelial dysfunction between Sepsis and its preceding risk factors (Aging and Chronic Disease). J Clin Med 7: 400, 2018.

27. Wu L, Walas S, Leung W, Sykes DB, Wu J, Lo EH and Lok J: Neuregulin $1-\beta$ decreases IL- $1 \beta$-induced neutrophil adhesion to human brain microvascular endothelial cells. Transl Stroke Res 6: 116-124, 2015.

28. Burger D and Touyz RM: Cellular biomarkers of endothelial health: Microparticles, endothelial progenitor cells, and circulating endothelial cells. J Am Soc Hypertens 6: 85-99, 2012.

29. Parodi EM and Kuhn B: Signalling between microvascular endothelium and cardiomyocytes through neuregulin. Cardiovasc Res 102: 194-204, 2014.

30. Hedhli N, Dobrucki LW, Kalinowski A, Zhuang ZW, Wu X, Russell RR III, Sinusas AJ and Russell KS: Endothelial-derived neuregulin is an important mediator of ischaemia-induced angiogenesis and arteriogenesis. Cardiovasc Res 93: 516-524, 2012.

31. Stefanou C, Karatzanos E, Mitsiou G, Psarra K, Angelopoulos E, Dimopoulos S, Gerovasili V, Boviatsis E, Routsi C and Nanas S: Neuromuscular electrical stimulation acutely mobilizes endothelial progenitor cells in critically ill patients with sepsis. Ann Intensive Care 6: 21, 2016.

32. Awada HK, Hwang MP and Wang Y: Towards comprehensive cardiac repair and regeneration after myocardial infarction: Aspects to consider and proteins to deliver. Biomaterials 82 94-112, 2016

33. Lemmens K, Segers VF, Demolder M and De Keulenaer GW: Role of neuregulin-1/ErbB2 signaling in endothelium-cardiomyocyte cross-talk. J Biol Chem 281: 19469-19477, 2006.

34. Wu C, Gui C, Li L, Pang Y, Tang Z and Wei J: Expression and secretion of neuregulin-1 in cardiac microvascular endothelial cells treated with angiogenic factors. Exp Ther Med 15 $3577-3581,2018$

35. Fernandes CJ Jr and de Assuncao MS: Myocardial dysfunction in sepsis: A large, unsolved puzzle. Crit Care Res Pract 2012: 896430, 2012.

36. Pathan N, Franklin JL, Eleftherohorinou H, Wright VJ, Hemingway CA, Waddell SJ, Griffiths M, Dennis JL, Relman DA Harding SE, et al: Myocardial depressant effects of interleukin 6 in meningococcal sepsis are regulated by p38 mitogen-activated protein kinase. Crit Care Med 39: 1692-1711, 2011.

37. Pathan N, Hemingway CA, Alizadeh AA, Stephens AC, Boldrick JC, Oragui EE, McCabe C, Welch SB, Whitney A, O'Gara P, et al: Role of interleukin 6 in myocardial dysfunction of meningococcal septic shock. Lancet 363: 203-209, 2004.

38. Waage A, Brandtzaeg P, Halstensen A, Kierulf P and Espevik T: The complex pattern of cytokines in serum from patients with meningococcal septic shock. Association between interleukin 6 , interleukin 1, and fatal outcome. J Exp Med 169: 333-338, 1989.

39. Leblais V, Jo SH, Chakir K, Maltsev V, Zheng M, Crow MT, Wang W, Lakatta EG and Xiao RP: Phosphatidylinositol 3-kinase offsets cAMP-mediated positive inotropic effect via inhibiting $\mathrm{Ca}^{2+}$ influx in cardiomyocytes. Circ Res 95: 1183-1190, 2004

40. Britsch S: The neuregulin-I/ErbB signaling system in development and disease. Adv Anat Embryol Cell Biol 190: 1-65, 2007.

41. Mencel M, Nash M and Jacobson C: Neuregulin upregulates microglial $\alpha 7$ nicotinic acetylcholine receptor expression in immortalized cell lines: Implications for regulating neuroinflammation. PLoS One 8: e70338, 2013.

42. Li Y, Lein PJ, Ford GD, Liu C, Stovall KC, White TE, Bruun DA, Tewolde T, Gates AS, Distel TJ, et al: Neuregulin-1 inhibits neuroinflammatory responses in a rat model of organophosphate-nerve agent-induced delayed neuronal injury. J Neuroinflammation 12 : $64,2015$.
43. Solomon W, Wilson NO, Anderson L, Pitts S, Patrickson J, Liu M, Ford BD and Stiles JK: Neuregulin-1 attenuates mortality associated with experimental cerebral malaria. J Neuroinflammation 11: 9, 2014

44. Gholami M, Mazaheri P, Mohamadi A, Dehpour T, Safari F, Hajizadeh S, Moore KP and Mani AR: Endotoxemia is associated with partial uncoupling of cardiac pacemaker from cholinergic neural control in rats. Shock 37: 219-227, 2012.

45. Fernandez R, Nardocci G, Navarro C, Reyes EP, Acuña-Castillo C and Cortes PP: Neural reflex regulation of systemic inflammation: Potential new targets for sepsis therapy. Front Physiol 5: 489, 2014

46. Rosas-Ballina M, Valdés-Ferrer SI, Dancho ME, Ochani M, Katz D, Cheng KF, Olofsson PS, Chavan SS, Al-Abed Y, Tracey KJ, et al: Xanomeline suppresses excessive pro-inflammatory cytokine responses through neural signal-mediated pathways and improves survival in lethal inflammation. Brain Behav Immun 44: 19-27, 2015.

47. Matsukawa R, Hirooka Y, Ito K, Honda N and Sunagawa K: Central neuregulin-1/ErbB signaling modulates cardiac function via sympathetic activity in pressure overload-induced heart failure. J Hypertens 32: 817-825, 2014.

48. Schmidt H, Müller-Werdan U, Hoffmann T, Francis DP, Piepoli MF, Rauchhaus M, Prondzinsky R, Loppnow H, Buerke M, Hoyer D, et al: Autonomic dysfunction predicts mortality in patients with multiple organ dysfunction syndrome of different age groups. Crit Care Med 33: 1994-2002, 2005.

49. Jabbour A, Gao L, Kwan J, Watson A, Sun L, Qiu MR, Liu X, Zhou MD, Graham RM, Hicks M, et al: A recombinant human neuregulin-1 peptide improves preservation of the rodent heart after prolonged hypothermic storage. Transplantation 91: 961-967, 2011.

50. Fattahi F, Kalbitz M, Malan EA, Abe E, Jajou L, Huber-Lang MS, Bosmann M, Russell MW, Zetoune FS, Ward PA: Complement-induced activation of MAPKs and Akt during sepsis: role in cardiac dysfunction. FASEB J 31: 4129-4139, 2017.

51. Checchia PA, Schierding W, Polpitiya A, Dixon D, Macmillan S, Muenzer J, Stromberg P, Coopersmith CM, Buchman TG and Cobb JP: Myocardial transcriptional profiles in a murine model of sepsis: Evidence for the importance of age. Pediatr Crit Care Med 9: 530-535, 2008

52. Zhou H, Qian J, Li C, Li J, Zhang X, Ding Z, Gao X, Han Z, Cheng Y and Liu L: Attenuation of cardiac dysfunction by HSPA12B in endotoxin-induced sepsis in mice through a PI3K-dependent mechanism. Cardiovasc Res 89: 109-118, 2011.

53. Cvijanovich N, Shanley TP, Lin R, Allen GL, Thomas NJ, Checchia P, Anas N, Freishtat RJ, Monaco M, Odoms K, et al: Genomics of Pediatric SIRS/Septic Shock Investigators: Validating the genomic signature of pediatric septic shock. Physiol Genomics 34: 127-134, 2008.

54. Preau S, Delguste F, Yu Y, Remy-Jouet I, Richard V, Saulnier F, Boulanger E and Neviere R: Endotoxemia engages the RhoA kinase pathway to impair cardiac function by altering cytoskeleton, mitochondrial fission, and autophagy. Antioxid Redox Signal 24: 529-542, 2016.

55. Ky B, Kimmel SE, Safa RN, Putt ME, Sweitzer NK, Fang JC, Sawyer DB and Cappola TP: Neuregulin-1 beta is associated with disease severity and adverse outcomes in chronic heart failure. Circulation 120: 310-317, 2009.

56. Odiete O, Hill MF and Sawyer DB: Neuregulin in cardiovascular development and disease. Circ Res 111: 1376-1385, 2012.

57. Chen XL, Xia ZF, Wei D, Han S, Ben DF and Wang GQ: Role of p38 mitogen-activated protein kinase in Kupffer cell secretion of the proinflammatory cytokines after burn trauma. Burns 29: 533-539, 2003.

58. Mockridge JW, Marber MS and Heads RJ: Activation of Akt during simulated ischemia/reperfusion in cardiac myocytes. Biochem Biophys Res Commun 270: 947-952, 2000.

59. Talmor D, Applebaum A, Rudich A, Shapira Y and Tirosh A: Activation of mitogen-activated protein kinases in human heart during cardiopulmonary bypass. Circ Res 86: 1004-1007, 2000.

60. Dong X, Liu Y, Du M, Wang Q, Yu CT and Fan X: P38 mitogen-activated protein kinase inhibition attenuates pulmonary inflammatory response in a rat cardiopulmonary bypass model. Eur J Cardiothorac Surg 30: 77-84, 2006.

61. Menon R and Papaconstantinou J: p38 Mitogen activated protein kinase (MAPK): A new therapeutic target for reducing the risk of adverse pregnancy outcomes. Expert Opin Ther Targets 20: $1397-1412,2016$. 
62. Antoon JW, Bratton MR, Guillot LM, Wadsworth S, Salvo VA Elliott S, McLachlan JA and Burow ME: Pharmacology and anti-tumor activity of RWJ67657, a novel inhibitor of p38 mitogen activated protein kinase. Am J Cancer Res 2: 446-458, 2012.

63. Kim SJ, Baek KS, Park HJ, Jung YH and Lee SM: Compound 9a, a novel synthetic histone deacetylase inhibitor, protects against septic injury in mice by suppressing MAPK signalling. Br J Pharmacol 173: 1045-1057, 2016.

64. Su J, Cui X, Li Y, Mani H, Ferreyra GA, Danner RL, Hsu LL, Fitz Y and Eichacker PQ: SB203580, a p38 inhibitor, improved cardiac function but worsened lung injury and survival during Escherichia coli pneumonia in mice. J Trauma 68: 1317-1327, 2010.

65. Wang J, Zhou J, Wang Y, Yang C, Fu M, Zhang J, Han X, $\mathrm{Li} \mathrm{Z}, \mathrm{Hu} \mathrm{K}$ and $\mathrm{Ge} \mathrm{J}$ : Qiliqiangxin protects against anoxic injury in cardiac microvascular endothelial cells via NRG-1/ ErbB-PI3K/Akt/mTOR pathway. J Cell Mol Med 21: 1905-1914, 2017.

66. Vessey DA, Li L and Kelley M: Ischemic preconditioning requires opening of pannexin-1/P2X(7) channels not only during preconditioning but again after index ischemia at full reperfusion. Mol Cell Biochem 351: 77-84, 2011.

67. Zhuo C, Wang Y, Wang X, Wang Y and Chen Y: Cardioprotection by ischemic postconditioning is abolished in depressed rats: Role of Akt and signal transducer and activator of transcription-3. Mol Cell Biochem 346: 39-47, 2011.

68. Kirabo A, Ryzhov S, Gupte M, Sengsayadeth S, Gumina RJ, Sawyer DB and Galindo CL: Neuregulin-1 $\beta$ induces proliferation, survival and paracrine signaling in normal human cardiac ventricular fibroblasts. J Mol Cell Cardiol 105: 59-69, 2017.

69. Bopassa JC, Ferrera R, Gateau-Roesch O, Couture-Lepetit E and Ovize M: PI 3-kinase regulates the mitochondrial transition pore in controlled reperfusion and postconditioning. Cardiovasc Res 69: 178-185, 2006.

70. Rahman S, Li J, Bopassa JC, Umar S, Iorga A, Partownavid P and Eghbali M: Phosphorylation of GSK-3 $\beta$ mediates intralipid-induced cardioprotection against ischemia/reperfusion injury. Anesthesiology 115: 242-253, 2011.

71. Guo LW, Gao L, Rothschild J, Su B and Gelman IH: Control of protein kinase $\mathrm{C}$ activity, phorbol ester-induced cytoskeletal remodeling, and cell survival signals by the scaffolding protein SSeCKS/GRAVIN/AKAP12. J Biol Chem 286: 38356-38366, 2011.

72. Siafakas NM, Antoniou KM and Tzortzaki EG: Role of angiogenesis and vascular remodeling in chronic obstructive pulmonary disease. Int J Chron Obstruct Pulmon Dis 2: 453-462, 2007.

73. Mount PF, Kemp BE and Power DA: Regulation of endothelial and myocardial NO synthesis by multi-site eNOS phosphorylation. J Mol Cell Cardiol 42: 271-279, 2007.

74. Sawada N and Liao JK: Targeting eNOS and beyond: Emerging heterogeneity of the role of endothelial Rho proteins in stroke protection. Expert Rev Neurother 9: 1171-1186, 2009.

75. Rana MK and Worthylake RA: Novel mechanism for negatively regulating Rho-kinase (ROCK) signaling through Coronin1B protein in neuregulin 1 (NRG-1)-induced tumor cell motility. J Biol Chem 287: 21836-21845, 2012.

76. van Nieuw Amerongen GP, Koolwijk P, Versteilen A and van Hinsbergh VW: Involvement of RhoA/Rho kinase signaling in VEGF-induced endothelial cell migration and angiogenesis in vitro. Arterioscler Thromb Vasc Biol 23: 211-217, 2003.

77. Anwar KN, Fazal F, Malik AB and Rahman A RhoA/Rho-associated kinase pathway selectively regulates thrombin-induced intercellular adhesion molecule-1 expression in endothelial cells via activation of I kappa B kinase beta and phosphorylation of RelA/p65. J Immunol 173: 6965-6972, 2004.

78. Chang W, Xie JF, Xu JY and Yang Y: Effect of levosimendan on mortality in severe sepsis and septic shock: A meta-analysis of randomised trials. BMJ Open 8: e019338, 2018.
79. Bonner JM and Boulianne GL: Diverse structures, functions and uses of FK506 binding proteins. Cell Signal 38: 97-105, 2017

80. Bruno F, Xavier W and Ricard F: Safety and pharmacodynamic activity of a novel TREM-1 pathway inhibitory peptide in septic shock patients: Phase IIa clinical trial results. Intensive Care Med Exp 6 (Suppl 1): 1-33, 2018.

81. Fang SJ, Wu XS, Han ZH, Zhang XX, Wang CM, Li XY, Lu LQ and Zhang JL: Neuregulin-1 preconditioning protects the heart against ischemia/reperfusion injury through a PI3K/Akt-dependent mechanism. Chin Med J (Engl) 123: 3597-3604, 2010.

82. Zhou Z, Guo F, Dou Y, Tang J and Huan J: Guanine nucleotide exchange factor-H1 signaling is involved in lipopolysaccharide-induced endothelial barrier dysfunction. Surgery 154: 621-631, 2013.

83. Liu X, Gu X, Li Z, Li X, Li H, Chang J, Chen P, Jin J, Xi B, Chen $\mathrm{D}$, et al: Neuregulin-1/erbB-activation improves cardiac function and survival in models of ischemic, dilated, and viral cardiomyopathy. J Am Coll Cardiol 48: 1438-1447, 2006.

84. Galindo CL, Kasasbeh E, Murphy A, Ryzhov S, Lenihan S, Ahmad FA, Williams P, Nunnally A, Adcock J, Song Y, et al: Anti-remodeling and anti-fibrotic effects of the neuregulin-1 $\beta$ glial growth factor 2 in a large animal model of heart failure. J Am Heart Assoc 4: e000528, 2015.

85. Bersell K, Arab S, Haring B and Kühn B: Neuregulin1/ErbB4 signaling induces cardiomyocyte proliferation and repair of heart injury. Cell 138: 257-270, 2009.

86. Sawyer DB, Zuppinger C, Miller TA, Eppenberger HM and Suter TM: Modulation of anthracycline-induced myofibrillar disarray in rat ventricular myocytes by neuregulin-1beta and anti-erbB2: Potential mechanism for trastuzumab-induced cardiotoxicity. Circulation 105: 1551-1554, 2002.

87. Grazette LP, Boecker W, Matsui T, Semigran M, Force TL, Hajjar RJ and Rosenzweig A: Inhibition of ErbB2 causes mitochondrial dysfunction in cardiomyocytes: Implications for herceptin-induced cardiomyopathy. J Am Coll Cardiol 44: 2231-2238, 2004.

88. Caillaud K, Boisseau N, Ennequin G, Chavanelle V, Etienne M, Li X, Denis P, Dardevet D, Lacampagne A and Sirvent P: Neuregulin 1 improves glucose tolerance in adult and old rats. Diabetes Metab 42: 96-104, 2016.

89. Wang X, Zhuo X, Gao J, Liu H, Lin F and Ma A: Neuregulin-1 $\beta$ partially improves cardiac function in volume-overload heart failure through regulation of abnormal calcium handing. Front Pharmacol 10: 616, 2019.

90. Wang X, Liu Z, Duan HN and Wang L: Therapeutic potential of neuregulin in cardiovascular system: Can we ignore the effects of neuregulin on electrophysiology? Mini Rev Med Chem 16: 867-871, 2016.

91. Jabbour A, Hayward CS, Keogh AM, Kotlyar E, McCrohon JA, England JF, Amor R, Liu X, Li XY, Zhou MD, et al: Parenteral administration of recombinant human neuregulin-1 to patients with stable chronic heart failure produces favourable acute and chronic haemodynamic responses. Eur J Heart Fail 13: 83-92, 2011.

92. Gao R, Zhang J, Cheng L, Wu X, Dong W, Yang X, Li T, Liu X, Xu Y, Li X, et al: A Phase II, randomized, double-blind, multicenter, based on standard therapy, placebo-controlled study of the efficacy and safety of recombinant human neuregulin-1 in patients with chronic heart failure. J Am Coll Cardiol 55: 1907-1914, 2010

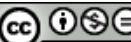

This work is licensed under a Creative Commons Attribution-NonCommercial-NoDerivatives 4.0 International (CC BY-NC-ND 4.0) License. 